\title{
Editorial
}

\section{Thermodilution in mechanically ventilated patients}

\author{
A. Versprille \\ Pathophysiological Laboratory, Department of Pulmonary Diseases, Erasmus University, Rotterdam, The Netherlands
}

Accepted: 14 October 1983

The advantage of a Swan-Ganz catheter is the possibility of measuring a diversity of variables including temperature in the pulmonary arterial blood for estimation of cardiac output by the thermodilution (TD) method.

The TD-method has several advantages over the Fick-method. Arterial and central venous blood do not have to be sampled, and oxygen consumption and carbon dioxide production do not have to be estimated. The method is fast, allows repetitive measurements and can be made operator proof, especially when the injections of cold indicator are done automatically $[1,2]$ and the analyses are performed online by a properly programmed cardiac output computer. However, during mechanical ventilation random observations have to be abandoned.

\section{Theoretical considerations}

Certain important conditions have to be fulfilled for the application of the TD-method: (a) complete mixing of indicator and blood [3]; (b) no loss of indicator between injection and sampling site [3]; (c) stationary blood flow during the period of the measurement $[2$, $4,5]$.

The third condition mentioned is not fulfilled during mechanical ventilation, when blood flow is nonstationary and fluctuates cyclically with the ventilation. I would therefore like to consider the consequences of this modulation for the reliability of the thermodilution measurements.

When flow is constant and the injections of "cold" are done as a delta function i.e. made within an infinitely short time, the area of the dilution curve can be estimated based on an exponential decay of its tail. When the injection takes a few seconds, and the rate of injection is not constant, an extrapolation of the curve based on an exponential function is inad- missible. In this situation the area has to be measured by planimetry, which can be done by computer sampling of the signal. When the flow is not constant, not only the semilog extrapolation but also planimetry fails to give an accurate estimation of mean cardiac output, because then the Stewart-Hamilton equation is no longer valid. This equation is essentially written as

$\overline{\mathrm{Q}}^{\prime}=\mathrm{m} / \int_{\mathrm{t}_{1}}^{\mathrm{t}_{2}} \Delta \mathrm{T}(\mathrm{t}) \cdot \mathrm{dt}$

where $\bar{Q}^{\prime}$ is mean cardiac output, $m$ is the amount of indicator and the integral function represents the area of the dilution curve, summing up all temperature changes $(\Delta T)$ in the blood during the dilution curve. This equation is derived from

$\mathrm{m}=\int_{\mathrm{t}_{1}}^{\mathrm{t}_{2}} \mathrm{Q}^{\prime}(\mathrm{t}) \cdot \Delta \mathrm{T}(\mathrm{t}) \cdot \mathrm{dt}$

where the total amount of indicator $(\mathrm{m})$ is equal to the sum of all temperature changes $(\Delta \mathrm{T})$ multiplied by the instantaneous flow values $\left(\mathrm{Q}^{\prime}\right)$ at the corresponding time moments $(\mathrm{t})$. Only when $\mathrm{Q}^{\prime}$ is constant can it be taken out of the integral function, because then all temperature changes are multiplied by the same value.

A reliable estimation of the area of the thermodilution curve needs the application of several corrections [2]. Periodic temperature changes in the baseline value, corresponding with the ventilatory cycle $[6-8]$, ascribed to changes in the ratio of venous return coming from the different regions, have to be analysed and extrapolated to the period of the dilution curve for correction of the area of the curve. Furthermore, long term trends in body-temperature have to be detected and extrapolated to the period of the curve, and the "leakage" of cold from the intra- 
corporeal part of the catheter after each injection has to be eliminated mathematically from each curve, when injection volume is corrected for catheter dead space. Rapid withdrawal of blood into the dead space [6] is not easily performed in fully automated observations.

\section{Experimental observations}

The nonstationary flow during mechanical ventilation has a characteristic pattern with a periodicity corresponding to the ventilatory cycle. During inflation a decrease in venous return and, therefore, in right ventricular output occurs, followed during early expiration by a fast recovery via an overshoot to an end expiratory plateau, as described by many authors [9] since the original description by Morgan et al. [10]. The variation in right ventricular stroke volume varied up to $400 \%$, inversely dependent on the level of mean flow [9].

Application of the TD-method under these circumstances with calculations based on the original Stewart-Hamilton equation, also resulted in a characteristic pattern of modulated flow estimates with a mean value of a series of 50 measurements, performed at each even percentage phase of the cycle, similar to the Fick values [2]. Thus, although the application of the Stewart-Hamilton equation is theoretically not allowed, in such a number of estimates the errors neutralize each other leading to a reliable mean value. The relative variation in the TD estimates around the mean was constant for different levels of mean flow and, thus, did not increase when flow fell, as the real modulation did. We hypothesized that the reason for this was a smoothing effect of the TD-method, because during a dilution curve the periods of high flow will have an increasing effect on the estimation and the periods of low flow a decreasing effect. Both effects will have been present, because the dilution curve took about $4 \mathrm{~s}$, which was a considerable part of the ventilatory cycle of $6 \mathrm{~s}$.

In spite of this smoothing effect we observed variations in TD-estimates between $60 \%$ and $130 \%$ of the mean under steady state circumstances. Thus, the estimations of cardiac output might have a difference of a factor of two, without a change in the condition of the animal, when observations are done randomly in the ventilatory cycle.

We also observed that the pattern of modulation shifted when mean flow decreased. This shift implies that during changing conditions not one moment in the ventilatory cycle will be found for the estimation of mean cardiac output, even when a systematic error is tolerated. Our results in pigs were recently con- firmed in dogs and in one patient, leading to the same conclusions [11].

An improvement to about $15 \%$ from the mean in the estimation of mean cardiac output was acquired by taking the mean of two estimates, obtained with a phase difference of half a ventilatory cycle [9]. A maximal reliability, with a deviation from the mean of about $2 \%$, was found when four estimates, equally spread over the ventilatory cycle, were averaged(J.R.C. Jansen, A. Versprille, unpublished work). Averaging a higher number of estimates did not improve the reliability. Thus, for estimation of mean cardiac output, four measurements at equally spread phases in the ventilatory cycle seem to be a minimal number for total neutralization of errors due to the application of the Stewart-Hamilton equation under non-stationary flow.

Estimation of cardiac output from measurements at preset phases in the ventilatory cycle requires a phase controlled injection of cold indicator, and therefore, a phase dependent signal from the ventilator via a control unit to an automatic injector. We developed this system respectively for a Starling ventilator [2], a computer controlled ventilator in the laboratory, and the Servo-ventilator (Siemens-Elema) in the clinical department.

\section{Clinical application}

The Swan-Ganz catheter with its long narrow tube for injection of cold fluid has several disadvantages for accurate estimation. Usually, the injections are done by hand. As a consequence the injection rate will not be constant, and the injection period of up to three seconds (also partly due to the large amount of injectate) certainly does not approximate to a delta function. Thus, a cardiac output computer programmed with an exponential extrapolation procedure will not estimate accurate curve areas. A computer programme based on a planimetric procedure will be feasible, but only when flow is constant and the corrections mentioned in the theoretical considerations are used in the calculations. Neglecting the condition of constant flow and using the Stewart-Hamilton equation, the longer duration of the injection will certainly contribute to the smoothing effect, as mentioned before, and will therefore lead to a smaller cyclic fluctuation in estimates of cardiac output than we found in our pig-studies [2].

However, due to a lack of accurate and systematic observations in mechanically ventilated patients we do not know how large the real errors of the estimates in patients are. The observation that repeated values are highly reproducible does not justify an assumption of accuracy. The estimations might have been 
performed at corresponding moments in the ventilatory cycle, e.g. when the noise of the ventilator or some other sign provides a reliable guide for the moment of injection. The widely used technique of injecting during the expiratory pause, whether or not prolonged, gives highly reproducible results but usually overestimates cardiac output.

For the moment, the best policy for obtaining an accurate value of mean cardiac output is the mean of a series of four TD-measurements spread equally in the ventilatory cycle. If fluid overload is a problem we may be compelled to use a smaller number of injections realising, however, that this is not the ideal.

\section{References}

1. Pfeiffer U, Birk M, Bluemel G (1979) Ein vollautomatischer Thermodilutions-Injektor. Biomedizinische Technik 24:60

2. Jansen JRC, Schreuder JJ, Bogaard JM, Rooyen W van, Versprille A (1981) Thermodilution technique for measurement of cardiac output during artificial ventilation. J Appl Physiol 51:584

3. Levett JM, Replogle RL (1979) Thermodilution cardiac output: a critical analysis and review of the literature. J Surg Res $27: 392$
4. Cropp JA, Burton AC (1966) Theoretical considerations and model experiments on the validity of indicator dilution methods for measurement of variable flow. Circ Res 18:26

5. Scheuer-Leeser M, Morquet A, Reul H, Inrich W (1977) Some aspects of the pulsation error in blood flow calculations by indicator-dilution technique. Med Biol Eng Comput 15:118

6. Ganz W, Swan HJC (1972) Measurement of blood flow by thermodilution. Am J Cardiol 29:241

7. Pavek E, Pavek K, Boschka D (1970) Mixing and observation errors in indicator dilution studies. J Appl Physiol 28:733

8. Saadjian A, Quercy JE, Torresani J (1976) Cardiac output measurement by thermodilution. Med Progr Technol 3:161

9. Versprille A, Jansen JRC, Schreuder JJ (1982) Dynamic aspects of the interaction between airway pressure and the circulation. In: Prakash O (ed) Applied physiology in clinical respiratory care. Martinus Nijhoff, The Hague, pp 447-463

10. Morgan BC, Martin WE, Hornbein TF, Crawford EW, Guntheroth WG (1966) Hemodynamic effects of intermittent positive pressure respiration. Anesthesiology 27:584

11. Snyder JV, Powner DJ (1982) Effects of mechanical ventilation on the measurement of cardiac output by thermodilution. Crit Care Med 10:677

Dr. Adrian Versprille

Pathophysiological Laboratory

Pulmonary Diseases Dept.

Erasmus University

Rotterdam

The Netherlands 